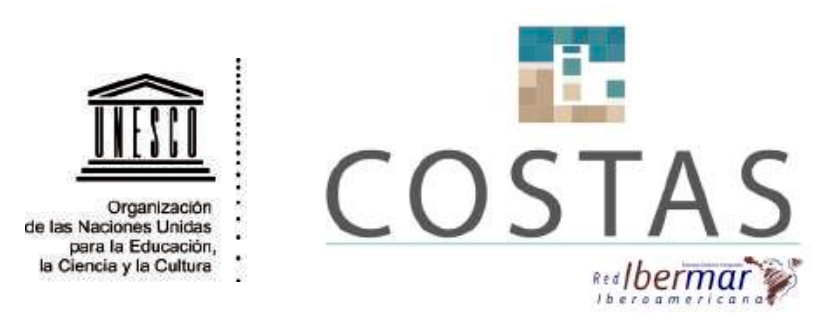

Enríquez-Hernández, G., Rosete-Vergés, F., RiveraArriaga E., 2020. Strengthening of intersectoral and intergovernmental relationships: learned lessons in the marine use planning process in Mexico. Revista Costas, 2(1): 169-184. doi: 10.26359/costas. 1502

Opinion Article / Artigo de Opinião / Artículo de Opinión

\section{Strengthening of Intersectoral and Intergovernmental Relationships: Learned Lessons in the Marine Use Planning Process in Mexico}

${ }^{1}$ Consultor Independiente

Omega 257, Romero de Terreros, 04310

Coyoacán, CDMX, México

${ }^{2}$ Escuela Nacional de Estudios Superiores-Unidad Morelia. Universidad Nacional Autónoma de México

Antigua Carretera a Pátzcuaro No. 8701.

Col. Ex Hacienda de San José de la Huerta. 58190

Morelia, Michoacán, México

e-mail: fernando.rosetev@enesmorelia.unam.mx

${ }^{3}$ Instituto EPOMEX-Universidad Autónoma de Campeche Campus 6, Héroe de Nacozari 480. 24029 Campeche,

Campeche, México

e-mail: evrivera@uacam.mx

Submitted: August 2020

Accepted: December 2020

Associate Editor: Eleonora Veron
Keywords: State government, federal government, marine use planning, integrated coastal zone management, policy instrument.

\begin{abstract}
Marine Use Planning (MUP) came up formally in Mexico as Environmental Policy Instrument in 1996. Through the National Environmental Policy for Sustainable Development of Oceans and Coast of Mexico issued in 2006, the Environmental and Natural Resources Secretariat (SEMARNAT) of Mexico encourages the formulation of Marine Use Plans. Considering the regional approach of this Plans, the relationships between stakeholders such as Federal and State Government (as public administration) and civil society, are essential in its formulation stages. Under this perspective and based on the learned experiences in the federal administrations (2000-2006 and 2006-2012), a non-exhaustive review on the way these
\end{abstract}




\section{COSTAS}

Revista Costas, Vol. 2 (2), 2020

relationships have been developed, and the main problems related to the coordination process between the involved sectors, is presented in this paper.

\section{Resumen}

El ordenamiento ecológico marino (OEM) es un instrumento de política ambiental que apareció formalmente en 1996. Con la publicación de la Política Ambiental Nacional para el Desarrollo Sustentable de Océanos y Costas de México en 2006, la Secretaría de Medio Ambiente y Recursos Naturales incremento la formulación de programas de OEM. Debido al carácter regional de los programas, las relaciones que se dan entre distintos actores de la administración pública federal, estatal y la sociedad civil, resultan fundamentales para su elaboración, por lo que en este trabajo se presenta una revisión no exhaustiva de cómo se han fomentado estas relaciones y las problemáticas asociadas a la coordinación entre los distintos sectores involucrados, a la luz de la experiencia adquirida en las administraciones federales (2000-2006 y 2006-2012).

Palabras clave: gobierno estatal, gobierno federal, ordenamiento ecológico marino, manejo integrado de zona costera, instrumento de política.

\section{Introducción}

A partir de la primera Conferencia de la Ley de los Mares de las Naciones Unidas que se realizó en 1958, hasta la firma de la Convención de las Naciones Unidas sobre el Derecho del Mar en 1982, se estableció un estatuto internacional con una norma de derecho positivo internacional para el uso y aprovechamiento de la zona marina de jurisdicción nacional (Rabasa, 1998). Esta convención se ha visto reflejada a través de la adopción de marcos jurídicos por parte de las naciones costeras como medio para ejercer sus derechos sobre la Zona Económica Exclusiva (ZEE) adyacente y creando así el nuevo régimen de los océanos (Division for Ocean Affairs and the Law of the Sea, 1993).

El establecimiento de la ZEE, ha permitido que los estados costeros tengan el control sobre el 35\% de la superficie del planeta, aunque la mayoría de ellos ha mostrado la falta de capacidades para resolver los múltiples conflictos que se presentan en esta zona (Miles, 1992). En el caso de México, al adoptar la ZEE y crear la Ley Federal del Mar las cuales entraron en vigor a partir de 1976, permitió que la nación tuviera acceso a los recursos renovables y no renova- bles que se encuentran dentro de un área marina de casi $3000000 \mathrm{de} \mathrm{km}^{2}$ (Rabasa, 1998).

En el capítulo 17 de la Agenda 21 sobre desarrollo sustentable, se propuso como parte de sus programas, la ordenación integral y el desarrollo sostenible de las zonas costeras y marinas, incluyendo la ZEE (ONU, Sin Fecha). Para atender este tema, México creó un instrumento denominado Ordenamiento Ecológico Marino (OEM) que dio la posibilidad de fomentar el desarrollo sustentable del océano, lo cual conlleva a la construcción de espacios de coordinación entre diferentes áreas y Órdenes de Gobierno (OG), para conocer y evaluar sus necesidades, así como sus problemáticas particulares.

Aunque el gobierno está obligado constitucional, política y moralmente a cumplir numerosas funciones y a ir tras la solución de un sinnúmero de problemas públicos, el ejercicio de su responsabilidad ocurre normalmente en modo desarticulado y hasta incoherente (Aguilar, 2011). En este sentido, la administración pública ha buscado a lo largo del tiempo, la manera más eficiente de realizar el trabajo colaborativo que se dan entre diferentes actores, nive- 
les y estructuras de gobierno (Conteh, 2013) y cuya complejidad requiere ser esclarecida para mejorar el desempeńo del aparato gubernamental.

En el caso de México, la relación que se da entre diferentes niveles de gobierno y el análisis de sus interacciones es reciente, debido en gran medida por la centralización de las acciones de gobierno (Ugalde, 2010), mientras que la relación entre diferentes áreas de la Administración Pública Federal (APF) se ha dado en el marco de comisiones intersecretariales que todavía hoy se presume como la mejor respuesta institucional al tema de la transversalidad (Ugalde, 2010).

Las fallas de coordinación entre sectores de la APF derivadas de sus políticas o programas particulares y entre OG, se debe a las interdependencias que existen para atender problemas que involucran a dos o más OG, las externalidades que generan las distintas actividades económicas y los conflictos derivados del uso de espacios o recursos. Estos tres elementos deben de negociarse de manera coherente y democrática a través de los mecanismos pertinentes, donde el resultado que se obtenga será en gran medida un reflejo de los estándares de transparencia, comunicación, cooperación y responsabilidad que predomine al interior del aparato gubernamental (Lerda et al., 2005).

Las formas de coordinación en el ámbito del OEM, adquiere un matiz muy diferente a los enfoques tradicionales en los que las relaciones intersectoriales e intergubernamentales se han aplicado para atender temas como la salud o la educación, ya que en el caso del OEM se trata de acordar el uso y protección de un espacio geográfico que por tradición se ha tratado en el escenario de la política nacional dentro de una agenda muy separada del resto de los problemas públicos.

Actualmente no existen análisis sobre los mecanismos de coordinación que se utilizaron en la elaboración de los OEM y por ende los autores realizaron una revisión empírica de sus experiencias más relevantes sobre las relaciones intersectoriales e intergubernamentales durante su participación en los procesos del Golfo de California y del Golfo de México y Mar Caribe, que se realizaron durante las administraciones federales (2000-2006 y 2006-2012).

\section{El instrumento de Ordenamiento Ecológico del Territorio (OET)}

Debido a la enorme complejidad del OEM, en la tabla 1 se presenta una síntesis en orden cronológico de los principales elementos jurídicos y administrativos que han permitido la aplicación del instrumento y los cuales se presentan con más detalle en las secciones 2 y 3 .

Lo que se conoce actualmente como el instrumento de Ordenamiento Ecológico del Territorio (OET), se inició como una propuesta hecha por la entonces Secretaría de Asentamientos Humanos y Obras Públicas durante la gestión 1976-1982, la cual estuvo dirigida a atender las demandas de la población relacionadas con el deterioro de los recursos naturales.
A partir de ese periodo, el OET ha transitado por diferentes etapas de desarrollo las cuales han sido ampliamente descritas por INE-SEMARNAP (2000), Azuela et al. (2006), Rosete-Vergés (2006), Rosete-Vergés et al. (2006) y Sánchez-Salazar (2013).

Como parte de esas etapas de desarrollo, el 13 de diciembre de 1996 apareció en el Diario Oficial de la Federación (DOF) el decreto de reforma de la Ley General del Equilibrio Ecológico y la Protección al Ambiente (LGEEPA) donde se creó la modalidad de OEM (INE-SEMARNAP, 2000) cuyo objeto es establecer los lineamientos y previsiones a que deberá sujetarse la preservación, restauración, protección y 


\section{COSTAS}

\begin{tabular}{|c|c|}
\hline Jurídicos & Administrativos \\
\hline $\begin{array}{l}\text { Ordenamiento ecológico del Territorio, propuesto por la } \\
\text { entonces Secretaría de Asentamientos Humanos y Obras } \\
\text { Públicas (1976-1982) }\end{array}$ & $\begin{array}{l}\text { Publicación del documento Sustentabilidad de los Océanos, } \\
\text { Políticas Nacionales e Internacionales (1999) }\end{array}$ \\
\hline $\begin{array}{l}\text { Decreto de reforma de la Ley General del Equilibrio Ecológico } \\
\text { y la Protección al Ambiente donde se crea la modalidad } \\
\text { de OEM (1996) }\end{array}$ & $\begin{array}{l}\text { Publicación de la Estrategia Ambiental para la Gestión } \\
\text { Integrada de la Zona Costera de México: retos } \\
\text { para el Desarrollo Sustentable (2000) }\end{array}$ \\
\hline $\begin{array}{l}\text { Elaboración y publicación del Reglamento de la Ley General } \\
\text { del Equilibrio Ecológico y la Protección al Ambiente } \\
\text { en materia de Ordenamiento Ecológico (2003) }\end{array}$ & $\begin{array}{l}\text { Publicación de la Política Ambiental Nacional para el } \\
\text { Desarrollo Sustentable de Océanos y Costas de México (2006) }\end{array}$ \\
\hline Decreto del Programa de OEM del Golfo de California (2006) & $\begin{array}{l}\text { Publicación de la Estrategia Nacional para el Ordenamiento } \\
\text { Ecológico del Territorio en Mares y Costas (2007) }\end{array}$ \\
\hline $\begin{array}{l}\text { Creación de la Comisión Intersecretarial para el Manejo } \\
\text { Sustentable de Mares y Costas (2008) }\end{array}$ & $\begin{array}{l}\text { En fase de elaboración la propuesta del programa de OEM } \\
\text { y regional del Pacífico Centor-Sur (2013) }\end{array}$ \\
\hline \multicolumn{2}{|l|}{$\begin{array}{l}\text { Decreto del Programa de OEM y Regional del Golfo } \\
\text { de México y Mar Caribe (2012) }\end{array}$} \\
\hline \multicolumn{2}{|l|}{$\begin{array}{l}\text { Decreto del Programa de OEM y Regional } \\
\text { del Pacífico Norte (2018) }\end{array}$} \\
\hline $\begin{array}{l}\text { Acuerdo mediante el cual se expidió la Política Nacional } \\
\text { de Mares y Costas (2018) }\end{array}$ & \\
\hline
\end{tabular}

aprovechamiento sustentable de los recursos naturales existentes en áreas o superficies específicas ubicadas en zonas marinas mexicanas, incluyendo las zonas federales adyacentes (DOF, 1996).

Con el inicio de la gestión 2001-2006 la Secretaría de Medio Ambiente y Recursos Naturales (SEMARNAT) promovió la elaboración y publicación del Reglamento de Ley General del Equilibrio Ecológico y Protección al Ambiente en Materia de Ordenamiento Ecológico (RLGEEPA-MOE), el cual permitió reglamentar las disposiciones de la LGEEPA en materia de OET, así como establecer las bases que deberán regir la actuación del gobierno federal durante el proceso de formulación de los OET, incluido el marino (DOF, 2003).
Con la existencia del RLGEEPA-MOE se contaba con la estructura administrativa y jurídica que permitió retomar el proceso que quedó inconcluso en el Golfo de California, y expedir el primer decreto de un programa de OEM en el 2006 (SEMARNAT, 2006a). Posteriormente, se continuo con la región del Golfo de México y Mar Caribe, donde se incluyó la parte terrestre a nivel regional dentro del proceso y cuyo decreto se emitió a finales de 2012 (DOF, 2012). Mientras que el acuerdo por el que se dio a conocer el Programa de Ordenamiento Ecológico Marino y Regional del Pacífico Norte (POEMR-PN), apareció en agosto del 2018 (DOF, 2018a), quedando pendiente la región del Pacífico Centro-Sur, la cual ya cuenta con avances en la elaboración de su programa (SEMARNAT-INECC, 2013). 


\section{Una política específica para océanos y costas}

En el caso de la gestión pública, el establecimiento de una política es un paso esencial, para definir espacios de acción donde el gobierno y los distintos actores se coordinan para lograr objetivos comunes, a través de estrategias específicas que involucran, entre otras cosas, instrumentos de gestión (Gil-Corrales, 2007), los cuales se encuentran definidos dentro de un marco jurídico que les proporciona certidumbre para su aplicación.

De acuerdo con Vallego (1992) la ausencia de una política de Estado para la zona oceánica se debe a dos factores (1) los asuntos relacionados con este ambiente no forman parte importante de la agenda política nacional, (2) tampoco acaparan la atención pública a menos de que se trate de un desastre ambiental o accidente. A pesar de lo anterior, resulta paradójico que con las características ambientales y oceanográficas de los océanos de México, no se contara con una política específica (Azuz et al., 2011).

En este sentido, los esfuerzos por establecer una política para el desarrollo sustentable de los océanos de México, surgen formalmente a partir de la postura que presentó en 1999 la entonces Secretaría de Medio Ambiente, Recursos Naturales y Pesca (SEMARNAP) en el documento México. Sustentabilidad de los Océanos, Políticas Nacionales e Internacionales, donde se describieron los avances de la gestión gubernamental por resolver los problemas de mayor impacto sobre los mares y los océanos (SEMARNAP, 1999).

Posteriormente en el 2000, la SEMARNAP propuso la Estrategia Ambiental para la Gestión Integrada de la Zona Costera de México: Retos para el Desarrollo Sustentable, cuyo objetivo era plantear una estrategia para la gestión integrada de la zona costera de México y formular una serie de recomendaciones de política sustentadas en un diagnóstico de la problemática que atañe a las zonas costeras del país y las causas que la provocan (SEMARNAP, 2000).

Estos documentos de análisis y orientación, comenzaron a demostrar el creciente interés por parte de la APF, los gobiernos estatales, los académicos, así como de otras instancias de la sociedad civil por contribuir al debate acerca de los problemas y propuestas para el desarrollo de la zona costera y oceánica. Pero no fue hasta el 2006 que se generó un escenario político adecuado para emprender acciones a nivel administrativo que posicionara el tema de planeación y gestión oceánica dentro de los temas de interés nacional, considerando para ello las recomendaciones realizadas por la Organización para la Cooperación y el Desarrollo Económico y el Consejo Consultivo de Desarrollo Sustentable, en materia de manejo integrado de zona costera (MIZC) (SEMARNAT, 2007; Azuz, et al., 2011).

La anterior dio por resultado la publicación en 2006 de la Política Ambiental Nacional para el Desarrollo Sustentable de Océanos y Costas de México (PANDSOC) $)^{1}$, la cual estableció los lineamientos generales de política pública del sector ambiental para la gestión integrada de la zona costera de México (SEMARNAT, 2006b), así como la publicación en el 2007 de la Estrategia Nacional para el Ordenamiento Ecológico del Territorio en Mares y Costas (ENOETMC), donde se establecieron las estrategias a seguir por los instrumentos de política ambiental en diferentes zonas del país (SEMARNAT, 2007).

En la PANDSOC se mencionó como parte de los lineamientos de la política ambiental para océanos y costas, al instrumento de OEM (SEMARNAT,

${ }^{1}$ En 2018, se generó el acuerdo mediante el cual se expidió la Política Nacional de Mares y Costas (DOF, 2018b), la cual sustituyó a la PANDSOC. 


\section{里 \\ COSTAS}

2006b), el cual tenía que cumplir con dos elementos para lograr su formulación e implementación: (1) Relaciones Intersectoriales: poder establecer un dialogo en igualdad de condiciones de negociación y poder político entre los sectores de protección ambiental, el pesquero, el marítimo portuario, extracción de hidrocarburos y el turístico marino, (2) Relaciones Intergubernamentales: establecer la comunicación y la coordinación necesaria del gobierno federal con los gobiernos estatales, para desarrollar programas de OEM que incluyan la parte terrestre que se encuentra más allá de los 20 metros de zona federal marítimo terrestre, con el fin de disminuir la presión de las actividades en tierra sobre la zona oceánica adyacente y generar propuestas regionales de planeación y gestión del territorio costero.

La construcción de los espacios para que se den de forma efectiva estos dos tipos de relaciones, representa un claro ejemplo de las herramientas con la que cuenta la administración pública en México para operativizar el esquema de coordinación horizontal y vertical que forma parte de las 5 fases que se requieren para un proceso MIZC (Barragán, 2020).

Es importante aclarar que a pesar de que la planeación del medio oceánico se ha convertido recientemente en un medio para la gestión de los espacios y recursos disponibles en esta zona (Díaz de León et al., 2010), lo anterior no significa que en el caso del OEM se pretendiera separar los procesos ecológicos, sociales y económicos que se desarrollan en la parte oceánica, de los de la parte terrestre y dejar de lado la gestión simultánea de ambos ambientes. A pesar de la distribución de competencias que existe en la aplicación de las distintas modalidades del OET, los acuerdos por los que se dieron a conocer el POEMRPN y el Programa de Ordenamiento Ecológico Marino y Regional del Golfo de México y Mar Caribe (POEMR-GMMC) abarcaron en su diseño ambos ambientes donde se involucró además a los sectores del área terrestre, para impulsar una participación democrática formal que permitiera el diálogo, el intercambio de información y la toma de decisiones a nivel regional, con los retos que significó para ese entonces trabajar con una gran número de actores y con grandes espacios oceánicos y terrestres.

Para generar la coordinación necesaria en la elaboración de los programas OEM (incluida la parte terrestre), se utilizaron los mecanismos señalados en la Ley Orgánica de la Administración Pública Federal (LOAPF) y en el RLGEEPA-MOE, por lo que en los siguientes apartados de este documento se presenta de manera no exhaustiva, un panorama de cómo se fomentó esa coordinación y las problemáticas asociadas.

\section{Fortalecimiento de relaciones intersectoriales (Transversalidad)}

Para entender como estos mecanismos han sido incorporados para fomentar las relaciones intersectoriales en el caso del OEM, es necesario describir primero los intentos del sector ambiental, por vincular su política específica con el resto de las políticas públicas, ya que es bajo el auspicio de esta área de gobierno donde se generaron los primeros esfuerzos de planeación y protección de la zona costera y oceánica.
A partir de la gestión 1994-2000, la entonces SEMARNAP incorporó los primeros esfuerzos de coordinación intersectorial dentro del marco de su programación sectorial (SEMARNAT, 2006c), para fortalecer la cooperación y la coordinación que se requiere en un esquema de planeación integral que permita gestionar el desarrollo de las actividades sociales y productivas de manera sustentable. 


\section{res COSTAS}

Durante la gestión 2001-2006, la SEMARNAT continuó con estos esfuerzos y realizó un proceso de concertación donde se incorporaron un total de 32 dependencias federales, con el fin de que incluyeran de manera voluntaria aquellos programas, acciones y proyectos prioritarios de su competencia, como parte del Programa para Promover el Desarrollo Sustentable en el Gobierno Federal, al cual se les daría seguimiento a través de las Agendas de Transversalidad de Políticas Públicas para el Desarrollo Sustentable (SEMARNAT, 2006c).

En la gestión 2007-2012, el Plan Nacional de Desarrollo estableció la Estrategia Integral de Política Pública (EIPP) como un mecanismo de coordinación entre las diferentes dependencias de la APF (DOF, 2007), lo cual permitió vincular tanto los 5 ejes de política como los objetivos nacionales, al Programa Sectorial de Medio Ambiente 2007-2012. En particular, para el tema de ordenamiento ecológico (contenido en el objetivo 5 dicho programa sectorial), se indicaron las dependencias de la APF con las que era necesario coordinarse para la formulación y seguimiento de los programas de OET (DOF, 2008a).

Además de la EIPP, la ENOETMC consideró necesario crear un organismo que permitiera articular la PANDSOC con el resto de las políticas públicas (SEMARNAT, 2007), mediante el mecanismo dispuesto en la LOAPF (Gil-Corrales, 2007; Gil-Corrales, 2008), lo cual permitió en el 2008 la creación de la Comisión Intersecretarial para el Manejo Sustentable de Mares y Costas (CIMARES) (DOF, 2008b) y cuyo objetivo es coordinar, en el ámbito de sus respectivas competencias, las acciones de las dependencias y entidades de la APF relativas a la formulación e instrumentación de las políticas nacionales para la planeación, ordenación y desarrollo sustentable de los mares y las costas del territorio nacional.

En aquel momento, la CIMARES se integró por 10 secretarias de la APF y otros 7 organismos como invitados permanentes, que se dividieron en dos sub- comisiones, las cuales conformaron 7 grupos de trabajo (figura 1) que atendían una agenda consensuada de 20 temas transversal de atención prioritaria (SEMARNAT, 2020).

La falta de coordinación en la APF es uno de los principales obstáculos para la aplicación eficiente de las funciones del Estado, tal y como fue documentado por Moreno et al. (2010), en un estudio sobre el análisis jurídico de la gestión pesquera en el área del Golfo de California, donde la Secretaría de Marina (SEMAR), la Comisión Nacional de Pesca y Acuacultura (CONAPESCA), la Procuraduría Federal de Protección al Ambiente (PROFEPA) y la Comisión Nacional de Áreas Naturales Protegidas (CONANP), que eran las únicas instancias encargadas de realizar acciones en materia de inspección y vigilancia en la parte oceánica, difícilmente programaban visitas conjuntas que permitan la sanción de ilícitos que vayan más allá del marco de competencia correspondiente a cada organismo.

Por otro lado, el caso del proceso de OEM ha identificado sectores cuyo desarrollo de actividades tiene una contribución preponderante en la economía local y nacional o cuentan con un apoyo social y político, que los coloca con ventaja con relación al resto de otros sectores, lo cual dificulta la coordinación durante el proceso de formulación del OEM.

Ejemplo de lo anterior, son las actividades de pesca que se realizan en el Golfo de California donde se registra la mayor producción del país tanto por los volúmenes aportados por pesquerías, como por el alto valor de mercado que alcanzan algunas de ellas, como el camarón (CONAPESCA, 2008). Estas actividades se distribuyen a lo largo del Golfo de California y coinciden con zonas donde el sector turismo o de protección al ambiente tiene actividades o intereses.

En el caso del Golfo de México, las actividades de explotación de hidrocarburos han requerido de la instalación de 238 plataformas, distribuidas en 2 regiones marinas y 4 activos integrales (PEMEX, 


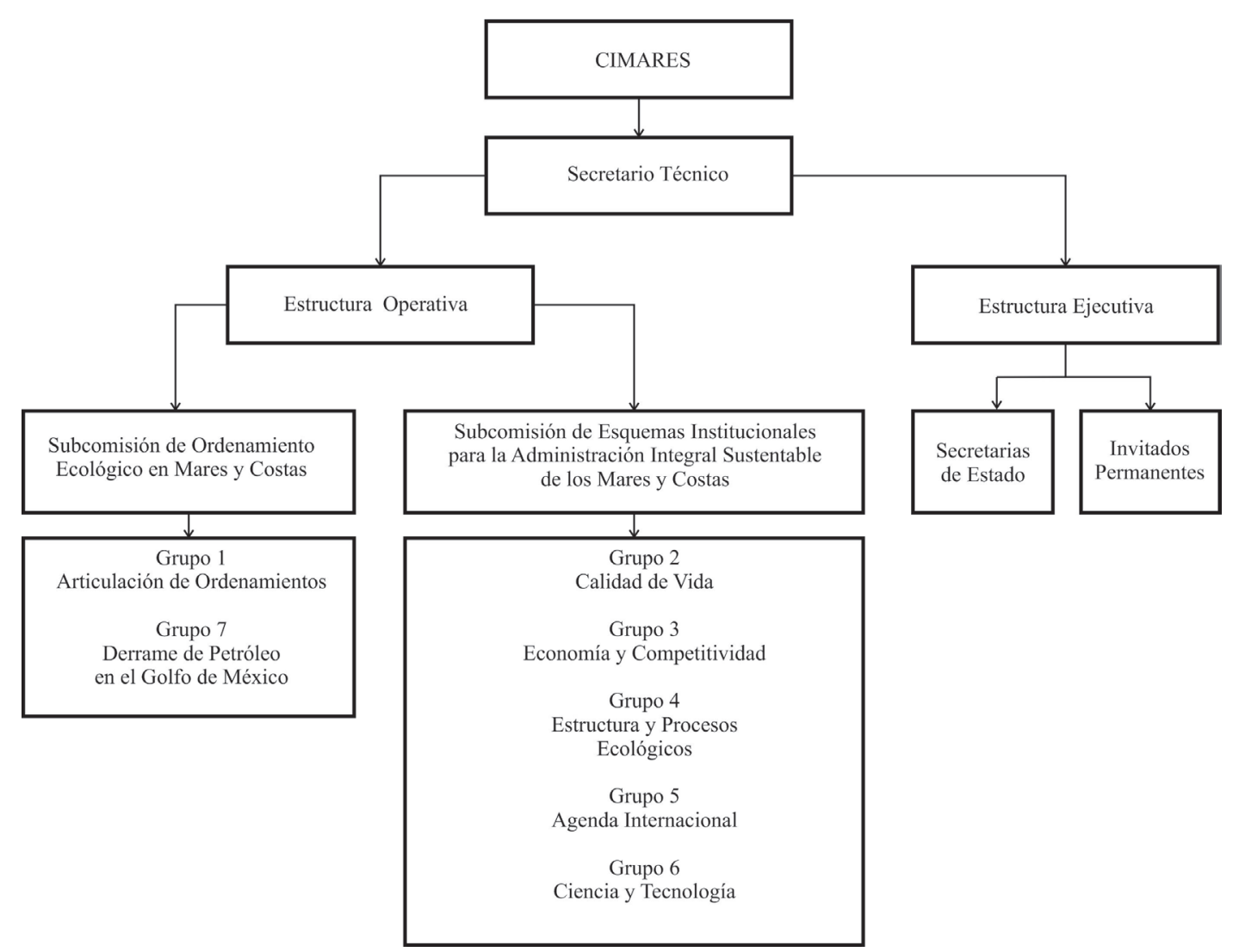

Figura 1. Estructura operativa y ejecutiva de la CIMARES (2012). Fuente: Modificado de SEMARNAT (2020).

Figure 1. CIMARES (2012). Operational and Executive Structure. Source: modified from SEMARNAT (2020).

2012) que coinciden con zonas donde el sector de pesca ribereńa y de protección al ambiente realizan actividades o también tienen intereses.

En los ejemplos que se mencionan sobre el Golfo de California y de México, muestran interacciones entre sectores que no siempre son positivas debido a la posición que se tiene con respecto a la apropiación del espacio geográfico que ocupan, los recursos que explotan y los beneficios económicos que se generan.

También fue posible identificar que el desarrollo de las actividades, presentan una marcada actuación sectorial como en el caso del programa de ordenamiento acuícola y pesquero que es promovido y sancionado por la CONAPESCA (Pedroza-Páez, 2011) y el instrumento de OEM. La aplicación de ambos instrumentos puede resultar legitima, pero es necesaria su articulación ya que el instrumento de OEM, apenas empezaba a encontrar su acomodo como una medida factible de desarrollo ante el resto del aparato gubernamental, por lo que la aparición de nuevos instrumentos que pudieran contraponerse a sus objetivos dificultarían su operación, con el riesgo de iniciar una competencia con el sector pesquero cuyo protagonismo es indudablemente mayor debido al peso político y social que históricamente representa.

Por otro lado, existen dependencias de la APF cuyas actividades se realizan únicamente en tierra y a pesar de que sus políticas particulares también tienen 


\section{res \\ COSTAS}

un efecto en la parte oceánica, por las prácticas de fomento que realizan, por los bienes, los servicios o las externalidades que generan, no buscaban como objetivo orientar el desarrollo desde un perspectiva territorial e integral, como es el caso de la Comisión Nacional Agropecuaria, la Secretaría de Economía o la Secretaria de Turismo, que mostraron tener dificultades para incorporarse a los procesos de OEM, para proponer estrategias que disminuyan la presión de las actividades sobre los ecosistemas costeros y el medio oceánico.
No obstante, también había dependencias de la APF como la entonces Secretaría de Desarrollo Social, la Secretaría de Comunicaciones y Transportes, la Comisión Federal de Electricidad y Petróleos Mexicanos cuya relación ambiente-territorio-sociedad estaba mejor definida dentro de sus labores cotidianas, lo cual fortaleció su actuación en el proceso, además de que tenían una participación constante en los diferentes comités de ordenamiento ecológico (COE) de los que formaron parte y que les permitió obtener experiencia sobre el proceso OEM.

\section{Fortalecimiento de las relaciones intergubernamentales}

Para articular un proceso de OEM es necesario considerar la delimitación del sistema de competencias que está establecido en la Constitución Política de los Estados Unidos Mexicanos, donde la parte oceánica (las aguas marinas interiores, el mar territorial, la zona contigua, la zona económica exclusiva y la plataforma continental) incluyendo las zonas federales adyacentes, están bajo la tutela de la federación, mientras que la parte terrestre incluye a los territorios de los estados, sus municipios y a diferentes regímenes de propiedad ${ }^{2}$.

En este sentido, la LOAPF tiene previsto un mecanismo de coordinación intergubernamental que también han sido incorporado al RLGEEPA-MOE, que consiste en la firma de un convenio de coordinación (Gil-Corrales, 2007; Gil-Corrales, 2008) con el que se pretendió formalizar la participación de las entidades federativas dentro del proceso de OEM y generar los compromisos institucionales que se requerían para la instalación de un COE (DOF, 2003).
Si bien las relaciones intergubernamentales están regidas por un marco de competencia definido a través de la legislación correspondiente para cada nivel de gobierno, la disposición que muestre cada uno por involucrarse en los procesos de OEM requiere de compromisos más allá de lo constitucionalmente establecido debido a que la complejidad de las problemáticas rebasa la capacidad de los OG para su atención de manera independiente (Hernández-Díaz, 2006).

Ejemplo de lo anterior, es el caso descrito por Pedroza-Páez (2011) donde la comunidad indígena Mayo solicitó a la SEMARNAT atender la problemática de contaminación y azolvamiento de la Bahía del Tóbari, que se ubica en el estado de Sonora. Esta problemática requería de la intervención de diversas dependencias federales y de la participación del gobierno estatal y municipal, por lo que se consideró necesario utilizar como mecanismo de coordinación al COE Marino del Golfo de California, a través de

\footnotetext{
${ }^{2}$ Es importante señalar que estas divisiones administrativas fuero consideradas para la definición de las diferentes modalidades de OET que se encuentran establecidas en la LGEEPA, por lo que fue necesario acordar en los convenios de coordinación que se firmaron con los distintos estados, el incluir dentro del proceso de OEM la presión generada por las actividades terrestres para cumplir con el esquema de lineamientos de política ambiental para océanos y costas propuesto en la PANDSOC (SEMARNAT, 2006b).
} 


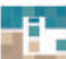 COSTAS}

la conformación de un grupo especial que atendió la solicitud de la comunidad Mayo.

Otra particularidad de las relaciones que surgen entre OG en el proceso de OEM es que se abordan también las problemáticas de la zona oceánica adyacente a los territorios estatales, por lo cual el compromiso de fomentar un cuidado de los recursos y espacios presentes en dicha zona, se puede ver como un elemento fuera de las atribuciones formales para los estados costeros, pero que debe ser atendido a través de la colaboración de los distintos OG incluyendo el orden municipal.

En este sentido, cuando se trata de los municipios con frente de costa o aquellos cuyas actividades económicas dependen de los bienes y servicios de la zona costera y oceánica, la situación cambia y se manifiesta una necesidad de controlar tanto las actividades que se dan en tierra como las que se dan en el ambiente oceánico.

Un ejemplo de lo anterior, fue la propuesta que realizó el municipio de Solidaridad en el estado de Quintana Roo, para la inserción de los arrecifes coralinos ubicados frente a las costas de este municipio, en el POEMR-GMMC, con el fin de proteger la diversidad física y biológica de la zona y fomentar el desarrollo sustentable de los recursos naturales presentes en el área, para que los servicios ambientales y de esparcimiento puedan ser aprovechados por los usuarios (Ayuntamiento del Municipio de Solidaridad, 2011).

La necesidad de realizar esta inserción, surge de la demanda de la sociedad local y de los contenidos del Programa Municipal de Desarrollo Urbano de Solidaridad (2010-2050), en el cual se estableció el preservar el medio ambiente, fomentar la conservación de los recursos naturales como cenotes, ríos subterráneos, flora, fauna terrestre y acuática, así como la conservación de arrecifes playas y dunas costeras (Ayuntamiento del Municipio de Solidaridad, 2011).
Revista Costas, Vol. 2 (2), 2020

Basado en lo anterior, el municipio realizó un esfuerzo para conseguir un consenso entre los diferentes sectores a nivel local y generó una propuesta técnica que consistió en una zonificación marina y una serie de criterios generales y específicos en los cuales se establece los usos, esperando que el COE aceptara el esquema de regulación.

Iniciativas de esta naturaleza resaltan la necesidad que existía por parte de ciertas administraciones municipales de articular procesos de planeación marina a nivel local para normar el uso de recursos y espacios, cuya competencia es federal, pero a través de los acuerdos pertinentes sería posible ofrecer un marco de regulación compartido.

Otro elemento clave en la elaboración de un programa de OEM fue la colaboración de los gobiernos estatales, cuyo trabajo se centró en participar activamente en los procesos, aportar información y validar los resultados que se obtuvieron durante la etapa de formulación. Mientras que la federación, se quedó con la labor de ejecutar y evaluar el instrumento, manteniendo con esto una visión de arriba-abajo que es característico de un federalismo aún centralizado (Cabrero-Mendoza, 2010; Jones et al., 2016) que tiende a debilitar, en este caso, las acciones por fortalecer la actuación de los estados y sus municipios dentro de los procesos de planeación regional.

En algunos casos, esta participación puede ser aún más limitada de acuerdo con las aportaciones reales que puedan hacer los funcionarios estatales durante los procesos y lo cual dependía de la experiencia que tuvieran en la elaboración de OET a nivel local o regional. Esto determinó de manera sustancial el grado de intervención de los estados que conformaron los diferentes COE en los procesos de OEM y las asimetrías en cuanto a conocimiento y experiencia fueron notorias, las cuales podían acentuarse, cuando las administraciones estatales en turno realizaban cambio de personal en áreas técnicas donde ya se contaba con 
experiencia y conocimiento, disminuyendo así las capacidades de estos gobiernos (Cabrero-Mendoza, 2004) para aportar a los procesos.

Desde la perspectiva de los estados costeros que participaron en los diferentes COE que se crearon durante la administración 2000-2006 y 2006-2012, los programas decretados debían facilitar la gestión de la zona oceánica, mientras que la aplicación de las estrategias y criterios ecológicos que se referían específicamente a procesos de planeación a través de distintos instrumentos de política, permitirían mejorar el desarrollo de la parte terrestre que correspondía a cada entidad federativa.

Desafortunadamente, no existen análisis de los impactos que tuvieron los programas de OEM, sobre la gestión y planeación de la zona terrestre de los estados costeros y sus municipios, pero en un estudio realizado por Nava-Fuentes et al. (2018), los autores presentaron una recopilación de las políticas públicas, el marco legal, las instituciones y las estrategias que aplican en el estado de Campeche para la planeación y gestión de la zona costera, incluyendo el POEMR-GMMC.

\section{COSTAS}

El estudio mencionado en el párrafo anterior hizo evidente la enorme complejidad administrativa que existe en el estado, donde el POEMR-GMMC forma parte de una serie de programas y estrategias que deben coexistir para lograr una planeación lo mejora articulada posible y cumplir con las metas, los objetivos y los plazos con los que fueron diseñados cada uno. La falta de una política costera explícita denota una visión fragmentada que origina que la administración pública del estado de Campeche, tenga deficiencias para atender las problemáticas asociadas al MIZC (Nava-Fuentes et al., 2018) y reproduce los problemas de diseño, colaboración y coordinación que se dan a nivel federal.

Es importante señalar que la capacidad de coordinación a nivel horizontal y vertical en el proceso de OEM, se logró de forma práctica con la figura COE, que es un mecanismo de coordinación que debía contar con un órgano ejecutivo y un órgano técnico (figura 2).

Cada órgano, estaba compuesto por representantes de la APF, estatal y de la sociedad civil que se conformó a su vez, por los enlaces del sector académico que

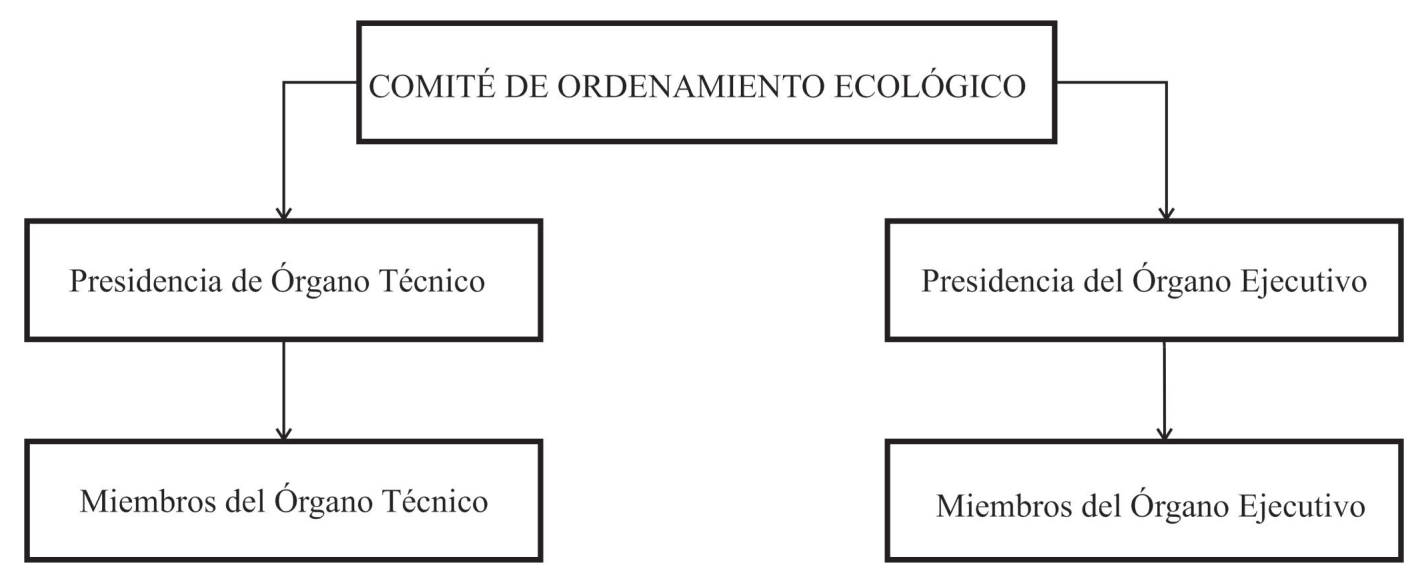

Figura 2. Estructura del Comité de Ordenamiento Ecológico Marino. Fuente: Elaboración propia a partir DOF (2003).

Figure 2. Structure of the Marine Use Planning Committee. 


\section{Fin COSTAS}

eran parte de los Consejo Consultivo para el Desarrollo Sustentable en cada estado participante y por representantes de los diferentes sectores productivos o sociales. Con esta estructura se pretendió legitimar

\section{Conclusiones}

Con esta revisión sobre el OEM, resultó evidente que el tratamiento que se la ha dado a las áreas oceánicas en México, no siguió en un principio la secuencia de pasos por los cuales toda problemática que requiere la intervención del Estado debe ser abordada y priorizada a través de políticas específicas, estrategias e instrumentos. Sin embargo, después de un largo proceso de modificaciones, reformas, creación de nuevos instrumentos y cambios en las prioridades en la política nacional, se concretó la estructura jurídica y administrativa con la que se cuenta actualmente para atender las posturas tanto nacionales como internacionales en materia de planeación y gestión de áreas oceánicas.

Considerando los logros obtenidos durante y posterior a las administraciones (2000-2006 y 20062012), México ya cuenta con tres elementos que de acuerdo con Chircop (1994) son necesarios para generar acciones reales de gobierno y los cuales son: (1) contar con una política nacional, (2) planear las actividades, (3) integrar la visión de diferentes sectores durante la aplicación de las estrategias. Es en este último punto, donde el fortalecimiento de las relaciones intersectoriales e intergubernamentales en el proceso de OEM mostró avances en el ámbito de la coordinación, que se vieron reflejados en la firma de tres decretos de OEM, como el caso del Golfo de California, el Pacífico Norte y el Golfo de México y Mar Caribe, pero cuya implementación está en peligro de no contar con el apoyo institucional para lograr los objetivos de gestión que fueron planteados en cada programa.
Revista Costas, Vol. 2 (2), 2020

y disminuir la exclusión de actores (Cabrero-Mendoza, 2000; Flannery et al., 2018) en los procesos de OEM.

Por otro lado, es importante observar que la integración a la que se refiere el inciso 3) del párrafo anterior, también podría considerar a los municipios costeros, como el caso de Solidaridad, ya que a la fecha los convenios de coordinación sólo involucran a las entidades federativas, por lo cual surge la pregunta de qué tan inclusivo debe ser el marco de la coordinación vertical para lograr los objetivos que se plantean en cada proceso de OEM, y ser más equitativos e incluyentes en la toma de decisiones.

No obstante, la inclusión de los municipios costeros como parte formal de los COE marino supera en mucho la capacidad de los mecanismos descritos para coordinar los procesos de OEM, por lo cual deberá intentarse nuevas fórmulas que permitan a la acción pública lograr una coordinación intergubernamental más incluyente, para contar con la participación de los gobiernos locales ya que los bienes y servicios ambientales de la zona costera y oceánica contribuyen, en algunos casos, de forma preponderante en la economía y a mejorar el bienestar de las comunidades locales.

La transición que experimentó México a partir de las situaciones planteadas en este documento, son recientes y por tanto, se cuenta con poca experiencia en la gestión de áreas oceánicas (León et al., 2004; Córdova et al., 2006), pero los avances logrados hablan del proceso de maduración que tuvo el aparato gubernamental y que requirió de un cambio en la forma en que se planteaba el desarrollo, al adoptar modelos de crecimiento sustentable, , ante una arraigada tradición sectorizada (Gil-Corrales, 2007). Lo 
anterior nos permite afirmar que la participación de varios actores para la planeación y gestión de la zona oceánica ya es una realidad, aunque es necesario afinar los procedimientos.

Este cambio hacia mejores acciones de coordinación y conciliación intersectorial e intergubernamental que se logró en las administraciones 2000-2006 y 2006-2012 también requiere de un mecanismo que permita la articulación competencial de todas las dependencias que conforman el COE, con el fin de sancionar el incumplimiento de las estrategias y criterios ecológicos contempladas en los programas de OEM, pues hasta ahora la relación que existe, se da a través de convenios de coordinación que carecen de obligaciones vinculantes (Domínguez, 2010).

También es necesario disminuir las contradicciones políticas y presupuestales en el área oceánica para que la aplicación de la política de mares y costas sea lo más consistente posible en la dimensión vertical y horizontal, por lo que habrá que tomar en cuenta

\section{Referencias}

Aguilar, LF. 2011. Políticas Públicas y Transversalidad, en: Ramos JM, Sosa J y Acosta F. (Coords.) La Evaluación de Políticas Públicas en México. Colegio de la Frontera Norte - INAP. Primera edición, México.

Ayuntamiento del Municipio de Solidaridad. 2011. Programa Municipal de Desarrollo Urbano de Solidaridad (2010-2050). Gobierno del Estado de Quintana Roo, México.

Azuela de la Cueva A, Cancino-Aguilar MÁ y Contreras-Morales C. 2006. El Ordenamiento Ecológico del Territorio en México: Génesis y Perspectiva. Secretaria de Medio Ambiente y Recursos Naturales, México.

Azuz I, Rivera-Arriaga E, Muñoz P y Ortega-Rubio A. 2011. Política Nacional para el Desarrollo Sustentable de Océanos y Costas en México: Génesis y Gestión. Región y Sociedad. 23(50): 279-289.

Barragán, JM. 2020. Progress of Coastal Management in Latin America and the Caribbean. Ocean and Coastal Management. 184: 105009. las relaciones asimétricas que se dan entre distintos sectores (Underdal, 1980) como puede ser el caso del sector pesca en relación con el sector conservación en el área del Golfo de California y tratar de disminuir las afectaciones.

Finalmente, el hacer operativos los conceptos de planeación integral a través del desarrollo de mecanismos que faciliten la transversalidad de las políticas públicas, mejoren las relaciones entre diferentes OG, promuevan la gestión simultanea de diferentes espacios competenciales y hagan en su conjunto más eficiente la aplicación de instrumentos de política ambiental como el OEM, ha resultado una tarea lenta y difícil a la luz de la experiencia adquirida en las administraciones federales 2000-2006 y 2006-2012. Ahora, la administración en turno (2018-2024), tiene la enorme labor de mantener los avances logrados y continuar con el fortalecimiento de la planeación y gestión oceánica en México.

Cabrero-Mendoza E. 2000. Usos y Costumbres de las Hechuras de las Políticas Públicas en México. Límites del Police Science en Contextos Culturales y Políticamente Diferentes. Gestión y Politica Pública. 9(2): 198-229.

Cabrero-Mendoza E. 2004. Capacidades Institucionales en Gobiernos Subnacionales de México ¿Un Obstáculo para la Descetralización Fiscal? Gestión y Politica Pública. 13(3): 753-784.

Cabrero-Mendoza E. 2010. Gobierno y Política Local en México: Luces y Sombras de las Reformas Descentralizadoras. Politica y Sociedad, 47(3), 165-186.

Chircop A. 1994. The Development of a National Ocean Policy and Institutional Implication. Foreing Relations Journal. 9(1): 48-62.

CONAPESCA (Comisión Nacional de Acuacultura y Pesca). 2008. Diagnóstico y Planificación Regional de la Pesca y Acuacultura. Región 1: Pacífico Norte. 


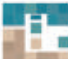 \\ COSTAS}

Programa Rector Nacional de Pesca y Acuacultura Sustentable", SAGARPA-CONAPESCA. México,

Conteh Ch. 2013. Strategic Inter-Organizational Cooperation in Complex Environments. Public Management Review. 15(4): 501-521.

Córdova-Vázquez A, Rosete-Vergés F, Enríquez-Hernández G. y Hernández de la Torre B. (Compiladores). 2006. Ordenamiento Ecológico Marino: Visión Temática de la Regionalización. Instituto Nacional de Ecología, México.

DOF (Diario Oficial de la Federación). 1996. Decreto que Reforma, Adiciona y Deroga Diversas Disposiciones de la Ley General del Equilibrio Ecológico y la Protección al Ambiente, Diario Oficial de la Federación, 13 de diciembre de 1996.

DOF (Diario Oficial de la Federación). 2003. Reglamento de la Ley General del Equilibrio Ecológico y Protección al Ambiente en Materia de Ordenamiento Ecológico, Diario Oficial de la Federación, 8 de agosto de 2003.

DOF (Diario Oficial de la Federación). 2007. Decreto por el que se Aprueba el Plan Nacional de Desarrollo 2007-2012, Diario Oficial de la Federación, 31 de mayo de 2007.

DOF (Diario Oficial de la Federación). 2008a. Decreto por el que se Aprueba el Programa Sectorial de Medio Ambiente y Recursos Naturales 2007-2012, Diario Oficial de la Federación, 21 de enero de 2008.

DOF (Diario Oficial de la Federación). 2008b. Acuerdo por el que se Crea con Carácter Permanente la Comisión Intersecretarial para el Manejo Sustentable de Mares y Costas, Diario Oficial de la Federación, 13 de junio de 2008.

DOF (Diario Oficial de la Federación). 2012. Acuerdo por el que se Expide la Parte Marina del Programa de Ordenamiento Ecológico Marino y Regional del Golfo de México y Mar Caribe y se da a Conocer la Parte Regional del Propio Programa, Diario oficial de la Federación, 24 de noviembre de 2012.

DOF (Diario Oficial de la Federación). 2018a. Acuerdo por el que se da a Conocer el Programa de Ordenamiento Ecológico Marino y Regional del Pacífico Norte, Diario Oficial de la Federación, 9 de agosto de 2018.

DOF (Diario Oficial de la Federación). 2018b. Acuerdo Mediante el Cual se Expide la Política Nacional de
Revista Costas, Vol. 2 (2), 2020

Mares y Costas de México, Diario Oficial de la Federación, 30 de noviembre de 2018.

Díaz de León A, Álvarez-Torres P e Iglesias-Barrón O. 2010. Experiencias Globales de Clasificación y Ejercicios de Zonificación Marina, en: Córdova-Vázquez A, Rosete-Vergés F, Enríquez-Hernández G y Hernández de la Torre B. (Compiladores), Ordenamiento Ecológico Marino. Visión Integrada de la Regionalización, Instituto Nacional de Ecología, México.

Division for Ocean Affairs and the Law of the Sea. 1993. Law and Order in the Oceans: United Nation Convention on the Law of the Sea, United Nations.

Domínguez J. 2010. Integralidad y Transversalidad de la Política Ambiental, en: Lezama JL y Graizbord B. (Coor.), Los Grandes Problemas de México IV Medio Ambiente, El Colegio de México, México.

Flannery W, Healy N and Luna M. 2018. Exclusion and Non-Participation in Marine Spatial Planning. $M a-$ rine Policy. 88: 32-40.

Gil-Corrales MA. 2007. Crónica Ambiental. Gestión Pública de Políticas Ambientales en México. Fondo de Cultura Económica - Secretaría de Medio Ambiente y Recursos Naturales - Instituto Nacional de Ecología, México.

Gil-Corrales MA. 2008. Políticas Públicas Orientadas al Medio Ambiente. Problemas de Integralidad, Integración y Congruencia. Informe Técnico. Instituto Nacional de Ecología, México.

Hernández-Díaz AM. 2006. Relaciones Intergubernamentales. Espacios Públicos. 9(18): 36-53.

INE-SEMARNAP (Instituto Nacional de Ecología - Secretaría de Medio Ambiente, Recursos Naturales y Pesca). 2000. El Ordenamiento Ecológico del Territorio: Logros y Retos del Desarrollo Sustentable 19952000, Instituto Nacional de Ecología, México.

Jones PJS, Lieberknecht LM and Qiu W. 2016. Marine Spatial Planning in Reality: Introduction to Cases Studies and Discussion of Findings. Marine Policy. 71: 256-264.

León C, Espejel I, Bravo LC, Fermán JL, Graizbord B, Sobrino LJ y Sosa J. 2004. El Ordenamiento Ecológico como un Instrumento de Política Pública para Impulsar el Desarrollo Sustentable: Caso en el Noroeste de México, en: Rivera-Arriaga E, Villalobos-Zapta G, Azuz-Adeath I y Rosado-May F. (Eds.), El Manejo Costero en México, Universidad Autónoma de 
Campeche-SEMARNAT- CETYS Universidad-Universidad de Quintana Roo, México.

Lerda JC, Acquatella J y Gómez JJ. 2005. Coordinación de Políticas Públicas: Desafíos y Oportunidades para una Agenda Fiscal-Ambiental, en: Acquatella J y Bárcena A (Eds.). Política Fiscal y Medio Ambiente. Bases para una Agenda Común. CEPAL.

Miles EL. 1992. Future Challenges in Ocean Management: Toward Integrated National Ocean Policy, en: Fabbri P. (Ed.), Ocean Management in Global Change. Elsevier Applied Science, New York.

Moreno J, Recio-Blanco X y Michel C. 2010. La Conservación del Acuario del Mundo. Un Análisis Jurídico de la Gestión Pesquera en el Golfo de California, Documento de Trabajo del Centro de Investigación y Docencia Económica (CIDE). División de Estudios Jurídicos. No. 46, México.

Nava-Fuentes JC, Arenas-Granados P and Cardoso-Martins F. 2018. Integrated Coastal Management in Campeche, Mexico; a Review After the Mexican Marine and Coastal National Policy. Ocean and Coastal Management. 154: 34-45.

ONU. Sin Fecha. Programa 21: Capítulo 17. Protección de los Océanos y de los Mares de Todo Tipo, Incluidos los Mares Cerrados y Semicerrados, y de las Zonas Costeras, y Protección, Utilización Racional y Desarrollo de sus Recursos Vivos. En: https://www.un.org/ spanish/esa/sustdev/agenda21/agenda21spchapter 17. htm Acceso: 4 de octubre de 2020.

Pedroza-Páez D. 2011. Análisis y Valoración del Manejo Sustentable de Mares y Costas. Resultados de un Análisis FODA y Recomendaciones para una Mejor Gestión Integral de la Zona Costera de México, en: Farinós Dasí J. (Editor y Coordinador), La Gestión Integrada de Zonas Costeras. ¿̇lgo Más que una Ordenación del Litoral Revisada? La GIZC Como Evolución de las Prácticas de Planificación y Gobernanza Territoriales. Universitat de València, España.

PEMEX (Petróleos Mexicanos). 2012. Anuario Estadístico 2012. Petróleos Mexicanos, México.

Rabasa E. 1998. La Zona Económica Exclusiva (Artículo 27, Párrafo octavo). Instituto de Investigaciones Jurídicas y Cámara de Diputados. (Eds.), Ochenta Años de Vida Constitucional en México. Instituto de Investigaciones Jurídicas y Cámara de Diputados, México.
Rosete-Vergés F. 2006. Semblanza Histórica del Ordenamiento Ecológico Territorial en México. Una Perspectiva Institucional, Secretaría de Medio Ambiente y Recursos Naturales - Instituto Nacional de Ecología, México.

Rosete-Vergés F, Enríquez-Hernández G y Córdova-Vázquez A. 2006. El Ordenamiento Ecológico Marino y Costero: Tendencias y Perspectivas. Gaceta Ecológica, 78: 67-83.

Sánchez-Salazar MT, Casado-Izquierdo JM y Bocco-Verdinelli G. 2013. La Política de Ordenamiento Territorial en México: de la Teoría a la Práctica. Reflexiones Sobre sus Avances y Retos a Futuro, en: Sánchez-Salazar MT, Bocco-Verdinelli, G y Casado-Izquierdo JM (Coor.), La Política de Ordenamiento Territorial en México: de la Teoría a la Práctica, UNAM-IGg, CIGA, SEMARNAT-INECC, México.

SEMARNAP (Secretaría de Medio Ambiente, Recursos Naturales y Pesca). 1999. México. Sustentabilidad de los Océanos, Políticas Nacionales e Internacionales, SEMARNAP, México.

SEMARNAP (Secretaría de Medio Ambiente, Recursos Naturales y Pesca). 2000. Estrategia Ambiental para la Gestión Integrada de la Zona Costera de México: Retos para el Desarrollo Sustentable, Instituto Nacional de Ecología-SEMARNAP, México.

SEMARNAT (Secretaría de Medio Ambiente y Recursos Naturales). 2006a. Programa de Ordenamiento Ecológico Marino del Golfo de California, SEMARNAT, México.

SEMARNAT (Secretaría de Medio Ambiente y Recursos Naturales). 2006b. Política Ambiental Nacional para el Desarrollo Sustentable de Océanos y Costas de México: Estrategia para su Conservación y Uso Sustentable, SEMARNAT, México.

SEMARNAT (Secretaría de Medio Ambiente y Recursos Naturales). 2006c. Memoria Documental para Promover el Desarrollo Sustentable en el Gobierno Federal 2001-2006 (PDS) y las Agendas de Transversalidad de Políticas Públicas para el Desarrollo Sustentable 2004-2006, Subsecretaría de Planeación y Política Ambiental-SEMARNAT, México.

SEMARNAT (Secretaría de Medio Ambiente y Recursos Naturales). 2007. Estrategia Nacional para el Ordenamiento Ecológico del Territorio en Mares y Costas, SEMARNAT, México. 


\section{rin \\ COSTAS}

SEMARNAT (Secretaría de Medio Ambiente y Recursos Naturales). 2020. Archivo Histórico de la CIMARES.

Antecedentes. en: https:/www.gob.mx/semarnat/ acciones-y-programas/comision-intersecretarial-parael-manejo-sustentable-de-mares-y-costas-cimares. Acceso: 12 de febrero de 2020.

SEMARNAT-INECC (Secretaría de Medio Ambiente y Recursos Naturales- Instituto Nacional de Ecología y Cambio Climático). 2013. Etapas de Caracterización y Diagnóstico del Estudio Técnico del Programa de Ordenamiento Marino y Regional del Pacífico Centro-Sur, UABC-IIO Centro Nacional de Datos Oceanográficos, México.
Revista Costas, Vol. 2 (2), 2020

Ugalde V. 2010. Distribución de Competencias y Relaciones Intergubernamentales en el Sistema Federal Mexicano, en: Méndez JL. (Coor.) Los Grandes Problemas de México XIII Políticas públicas, El Colegio de México, México.

Underdal A. 1980. Integrated Marine Policy: What? Why? How? Marine Policy. 169-180

Vallego SM. 1992. Integrated Marine Policies: Goals and Constrains, en: Fabbri P. (Ed.), Ocean Management in Global Change. Elsevier Applied Science, New York. 